\title{
Imaging of appendicitis
}

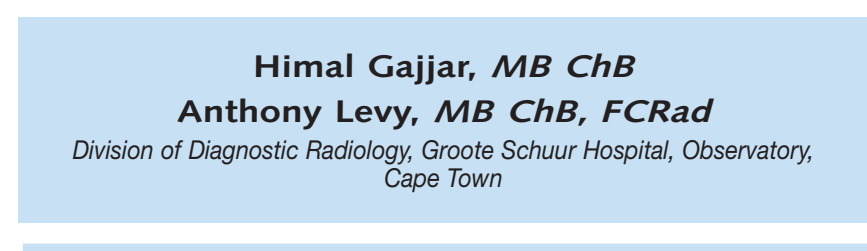

\section{Introduction}

Appendicitis is one of the most common causes of abdominal pain requiring surgery. Early diagnosis and management are essential to reduce morbidity and mortality. Imaging is valuable in the diagnosis of cases that are clinically atypical. Imaging also allows evaluation of the complications of appendicitis, which include perforation, peri-appendiceal abscess formation and portal pyaemia. In certain circumstances, conservative treatment of complicated appendicitis with percutaneous drainage is appropriate.

\section{Case 1}

A 62-year-old man with no past medical history of note presented to a secondary level hospital with a 5-day history of severe abdominal pain more severe on the right, nausea, vomiting and diarrhoea. The patient consulted 2 general practitioners prior to presentation, and analgesia

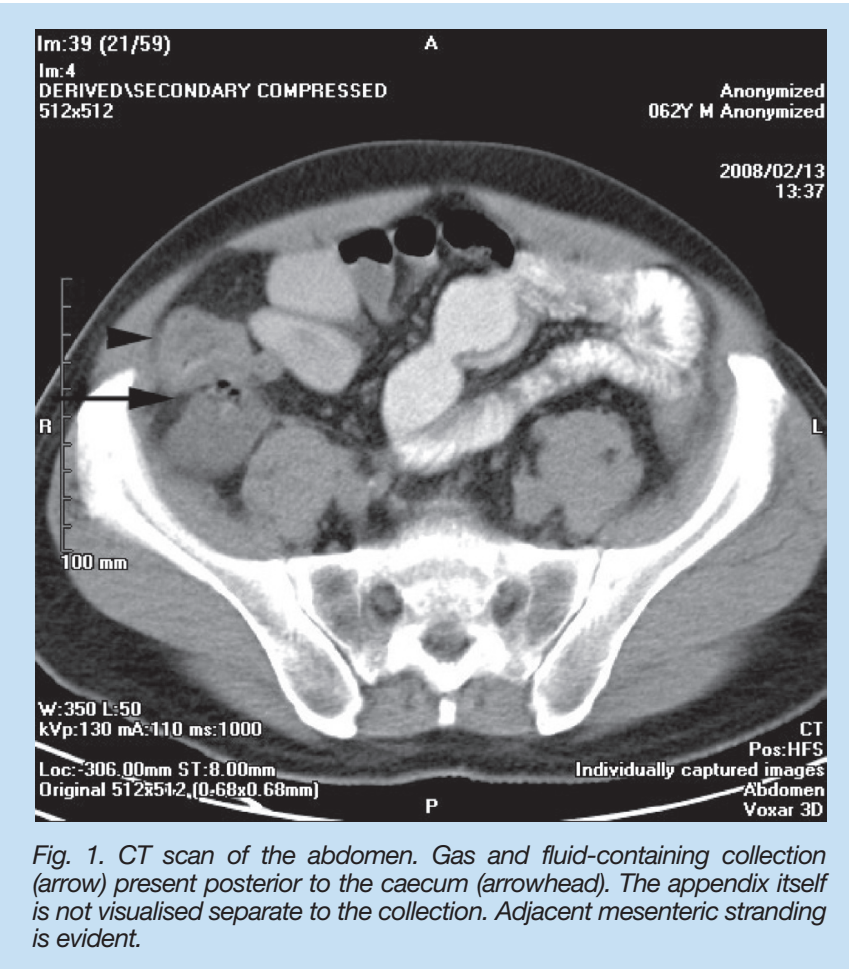

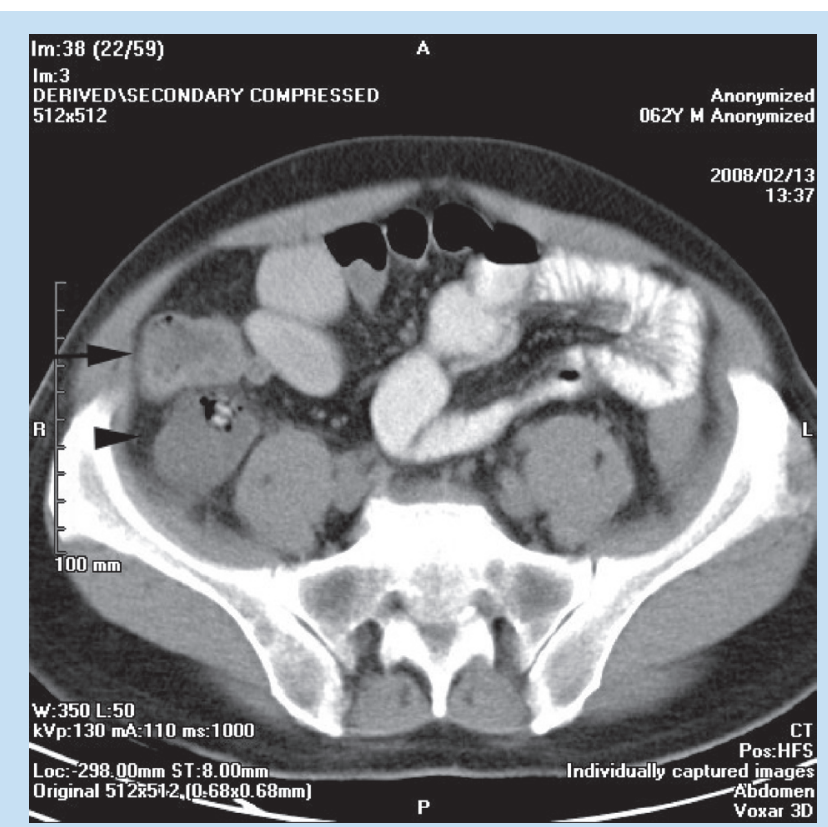

Fig. 2. CT scan of the abdomen. Retrocaecal collection (arrowhead) containing a high-density calculus consistent with an appendicolith. The caecum is indicated by the arrow. Fig. 2. is superior to Fig. 1.

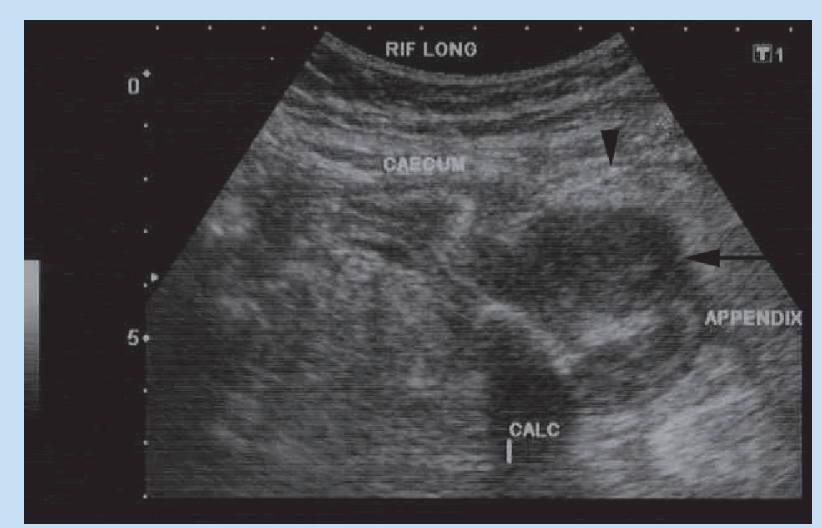

Fig. 3. Ultrasound of the abdomen. Distended appendix containing appendicolith. Adjacent low echogenicity fluid collection (arrow) and echogenic inflamed fat (arrowhead). Although not indicated in the figure, the appendiceal diameter was $12 \mathrm{~mm}$.

and antibiotics were prescribed. On examination, he was dehydrated with a tachycardia of $125 \mathrm{bpm}$ and a temperature of $37.9^{\circ} \mathrm{C}$. The abdomen was distended and diffusely tender, with guarding on the right side. No rebound was elicited. Dilated loops of small bowel were visible on the abdominal radiograph. White blood cell count was normal. Renal failure was evident with urea $=28 \mathrm{mmol} / \mathrm{l}$ and creatinine $=342 \mu \mathrm{mol} / \mathrm{l}$. The differential diagnosis included appendicitis, diverticulitis or perforated colonic neoplasia. The patient was initially treated with intravenous (IV) antibiotics and fluid, with some improvement in symptoms. 

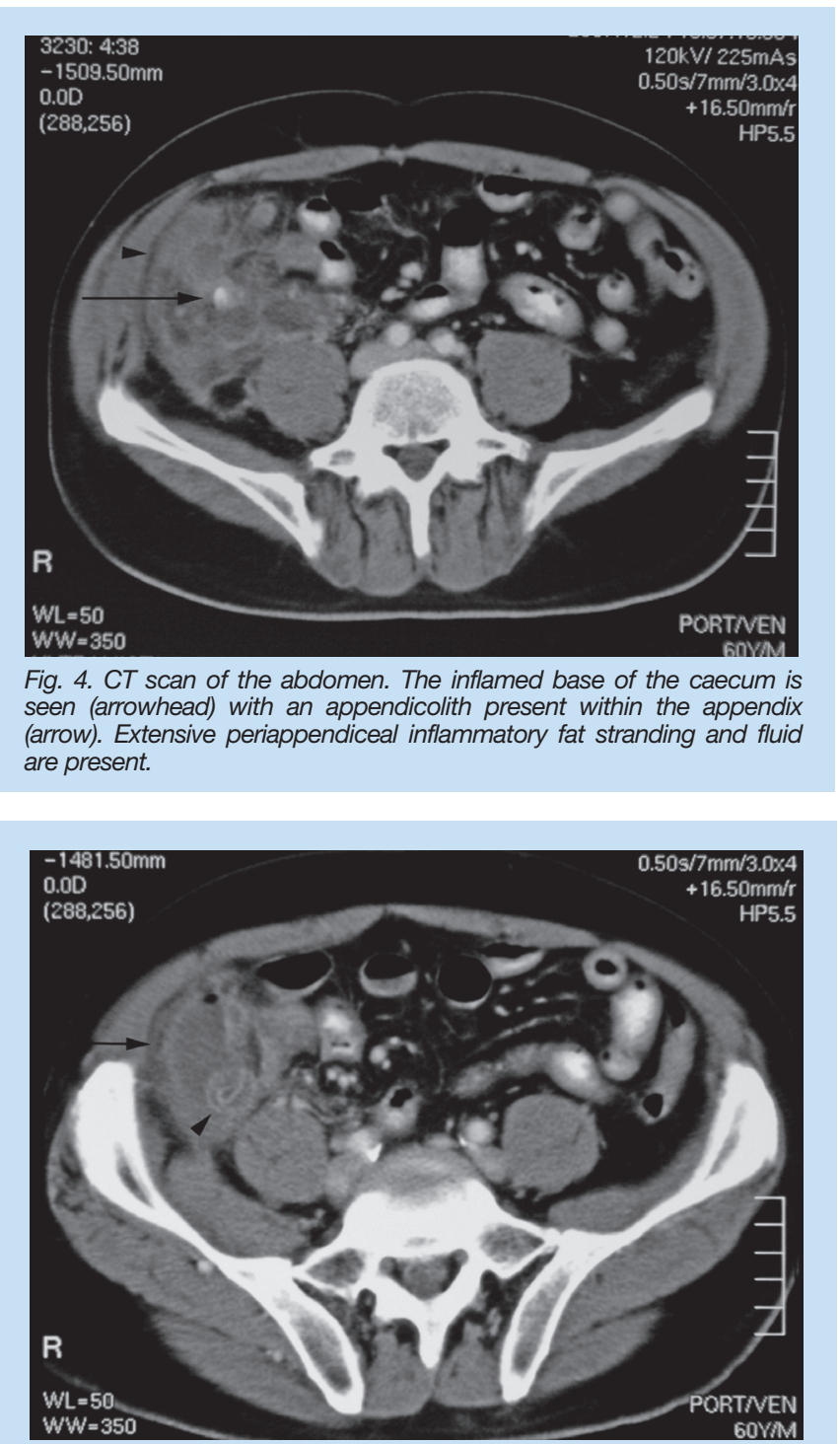

Fig. 5. CT scan of the abdomen. A fluid collection containing a gas locule (arrow) is seen adjacent to the thick-walled distended appendix (arrowhead). Fig. 5 is inferior to Fig. 4.

An abdominal ultrasound performed 3 days later was considered normal. Owing to lack of resolution of symptoms, a computed tomo graphy (CT) scan of the abdomen was performed with oral contrast. This revealed a retrocaecal gas and fluid collection (Fig. 1) containing an appendicolith (Fig. 2) which extended from the expected origin of the appendix to the subhepatic space. A diagnosis of peri-appendiceal abscess formation was made. The patient was treated with percutaneous drainage with a good result.

\section{Case 2}

A 60-year-old man with a background of type 2 diabetes mellitus presented to Groote Schuur Hospital, complaining of a 1-week history of right iliac fossa pain and diarrhoea. Four days prior to this presentation, he was assessed as having gastroenteritis and treated with intravenous rehydration. On examination he was apyrexial, with a tachycardia of $100 \mathrm{bpm}$. A tender mass was present in the right iliac fossa. Laboratory investigations revealed a raised white cell count of $13.9 \times 10^{9} / 1$.
On ultrasound imaging, a distended non-compressible appendix was present, containing an appendicolith (Fig. 3). An adjacent fluid collection was present with surrounding inflammatory fat stranding. A CT scan with oral and intravenous contrast was performed to delineate the extent of the collection. Again, the appendicolith (Fig. 4) was well seen, with the distended appendix present inferior to this (Fig. 5). The patient was treated with percutaneous drainage with a good clinical result. A colonoscopy excluded a caecal neoplasm as a cause for appendiceal obstruction.

\section{Discussion}

Acute appendicitis is one of the most common surgical emergencies in both the adult and paediatric populations. It occurs when the appendiceal lumen is obstructed, resulting in accumulation of fluid and subsequent inflammation and infection. The inflammation can progress, with resultant appendiceal rupture. ${ }^{1}$ Delayed diagnosis has serious consequences. These include appendiceal rupture, peri-appendiceal abscess formation, peritonitis and death. ${ }^{2}$ Traditionally, the diagnosis has been based on clinical assessment, with a reported accuracy of $71-97 \%{ }^{3}$ Owing to the dire consequences of appendiceal perforation, a negative laparotomy rate of $20 \%$ has been regarded as acceptable. ${ }^{4}$ Imaging can assist in the diagnosis of appendicitis and is usually indicated when the clinical signs are atypical. The goal of imaging is to facilitate the diagnosis of appendicitis or suggest an alternative diagnosis without delaying management. Imaging can also be used to evaluate complications of appendicitis and to guide percutaneous intervention.

Traditionally, plain film radiography and barium enema were the main imaging modalities used. However, owing to insensitivity and lack of specificity, these are no longer utilised specifically in the diagnosis of appendicitis.

Ultrasound evaluation of appendicitis was first described by Puylaert in $1986 .{ }^{5}$ He described a technique termed 'graded compression ultrasound. This involved using a high-frequency linear probe for imaging which provides excellent resolution but poor penetration of tissues. The patient is initially asked to localise the site of maximal tenderness, allowing a more focused ultrasonic examination. Gradual compression of the right iliac fossa both displaces overlying small-bowel loops which may obscure visualisation and brings the probe closer to the appendix. The compression is usually well tolerated. It is important to visualise the entire appendix as isolated appendicitis of the appendix tip is well described. ${ }^{2}$ A non-compressible appendix with a diameter $>6 \mathrm{~mm}$ confirms appendicitis. Echogenic inflamed surrounding mesenteric fat and the presence of an appendicolith strengthens diagnostic certainty.

In a meta-analysis conducted between 1986 and 1994, Orr et al. reported a sensitivity of $85 \%$ and a specificity of $92 \%$ among adult and paediatric populations. ${ }^{6}$ Sonography has the advantage of being quick to perform, without any need for patient preparation. Mobile examinations are possible. The most important advantage is the absence of ionising radiation in imaging, which has resulted in ultrasound being the initial imaging choice for appendicitis in many centres, especially in paediatric and pregnant patients. Evaluation of pelvic structures can also be performed in females, as gynaecological pathology is an important differential in the diagnosis of appendicitis. Unfortunately, ultrasound is highly operator-dependent and, even with good technique, the appendix may not be visualised. Appendiceal perforation may also result in false-nega- 
tive diagnoses, with relief of appendiceal obstruction resulting in loss of luminal distention and incompressibility.

CT scanning has emerged as a valuable resource in the imaging of appendicitis. This modality has the advantage of improved rates of visualisation of the appendix and surrounding structures, as well as more accurate evaluation of the rest of the abdomen. It allows more confident detection of a normal appendix, so excluding appendicitis as a cause of symptoms. Reported sensitivities and specificities in the diagnosis of appendicitis are between $88-100 \%$ and $91-99 \%$ respectively. ${ }^{1}$ Modern multidetector CT scanners allow rapid evaluation of the entire abdomen with excellent resolution both in the axial as well as sagittal and coronal planes. The biggest disadvantage is the high ionising radiation dose delivered, although techniques to reduce the dose have been developed.

The technique of examination varies, as many options are available. The entire abdomen can be imaged or the examination can be focused onto the pelvis. A focused examination reduces dose, at the risk of missing pathology in the rest of the abdomen. The scan may be performed with or without intravenous contrast. Withholding intravenous contrast reduces the risk of contrast media reactions and cost. However, intravenous contrast assists in evaluation of subtle cases and complications of appendicitis, such as portal pyaemia. Oral contrast media predominantly opacify the small bowel and improve detection of intra-abdominal collections. Unfortunately, oral contrast administration means delaying imaging to allow contrast media to transit the small bowel. Rectal contrast is extremely useful in evaluation of appendicitis as caecal distention is a prerequisite for some of the radiological signs discussed below. ${ }^{1}$

The main diagnostic criteria for appendicitis on $\mathrm{CT}$ are based on the morphology of the appendix, the caecum and the periappendiceal structures. Distention of the appendix to a diameter of $\geq 10 \mathrm{~mm}$, wall thickening $(>3 \mathrm{~mm})$ and wall hyperenhancement are useful diagnostic signs. An appendiceal diameter of $7-10 \mathrm{~mm}$ is regarded as inconclusive. The diameter threshold is larger than on ultrasound, and this is related to the lack of compression during CT scanning. Appendicoliths are not diagnostic of appendicitis but may have prognostic importance as a higher rate of perforation is found in their presence. Caecal changes relate to inflammation at the appendiceal opening at the base of the caecum. The result is caecal thickening; a number of signs related to this have been described. Peri-appendiceal inflammatory change manifests as fat stranding or a 'misty' appearance to the adjacent fat. The inflammatory change can also affect adjacent structures such as adjacent small bowel or the bladder. ${ }^{1}$ Unfortunately, as with ultrasound, CT is not a sensitive modality in detecting perforation. The findings of free air and periappendiceal collections are highly specific but have poor sensitivity?

Various alternate diagnoses can be suggested by CT scanning; these include: mesenteric adenitis, epiploic appendagitis, diverticulitis, Crohn's disease and caecal carcinoma.

The role of magnetic resonance imaging (MRI) in the imaging of appendicitis is currently being evaluated. It has the advantages of not utilising ionising radiation, and of having superior soft-tissue contrast and acquiring in direct multiplanar imaging. Unfortunately, MRI is more expensive with reduced availability in comparison with either CT or ultrasound; it is also unsuitable for imaging unstable patients owing to long acquisition times and the necessity for MRI-compatible monitoring equipment. It may have a role in the imaging of appendicitis in pregnant or paediatric patients in whom ultrasound is not conclusive.,

The radiology service can also provide a therapeutic option in specific situations. Percutaneous catheter drainage has shown its value in intra-abdominal abscess drainage in a wide variety of situations. It is a safe technique with good clinical results if utilised appropriately. Patients with localised, well-defined peri-appendiceal abscesses $>3 \mathrm{~cm}$ without generalised peritonitis are good candidates for percutaneous drainage; this may be performed under ultrasound or CT guidance with local anaesthetic and conscious sedation. Percutaneous drainage has been shown to reduce complications and hospital stay in comparison with open surgical drainage. Fistulous connections from the caecum to the abscess cavity may be demonstrated in a significant proportion of patients; however, in the vast majority, this connection closes within 2 weeks. In the vast majority of patients, the abscess resolves and interval appendicectomy may be performed electively. ${ }^{10,11}$

\section{Conclusion}

Imaging can provide assistance in the diagnosis of appendicitis and the complications thereof or suggest an alternative diagnosis. Choosing the appropriate imaging modality should be done on an individual case basis in discussion with the radiology service. In selected patients, therapeutic intervention in the form of percutaneous catheter drainage is appropriate.

1. Pinto Leite N, Pereira J, Cunha R, Pinto P, Sirlin C. CT evaluation of appendicitis and its complications: imaging and key diagnostic findings. Am J Roentgenol 2005; 185: 406-407.

2. Sivit C, Siegel M, Appelgate K, Newman K. When appendicitis is suspected in children. Radiographics 2001; 21: 247-262.

3. John H, Neff U, Kelemen M. Appendicitis diagnosis today: clinical and ultrasonic deductions. World J Surg 1993; 17: 243-249.

4. Jones PF. Suspected acute appendicitis: trends in management over 30 years. Br J Surg 2001; 88: 15701577.

5. Puylaert J. Acute appendicitis: US evaluation using graded compression. Radiology 1986; 158: 355-360.

6. Orr RK, Porter D, Hartman D. Ultrasonography to evaluate adults for appendicitis: decision making based on meta-analysis and probablistic reasoning. Acad Emerg Med 1995; 2: 644-650.

7. Bixby S, Lucey B, Soto J, Theysohn J, Ozonoff A, Varghese J. Perforated versus nonperforated acute appendicitis: accuracy of multidetector CT detection. Radiology 2006; 241(3): 780-786.

8. Singh A, Danrad R, Hahn P, Blake M, Mueller P, Novelline R. MR imaging of the acute abdomen and pelvis: acute appendicitis and beyond. Radiographics 2007; 27: 1419-1431.

9. Pedrosa I, Levine D, Eyvazzadeh A, Slewart B, Ngo L, Rofsky N. MR imaging evaluation of acute appendicitis in pregnancy. Radiology 2006; 238(3): 891-899.

10. Brooke JR, Ferderle M, Tolentino C. Periappendiceal inflammatory masses: CT directed management and clinical outcomes in 70 patients. Radiology 1988; 167: 13-16.

11. van Sonnenberg E, Wittich G, Casola G, et al. Periappendiceal abscess percutaneous drainage. Radiology 1987; 163: 23-26. 